\title{
Induction Heating Appliance with a Mobile Double-Coil Inductor
}

\author{
F. Sanz, Student Member, IEEE, C. Sagues, Senior Member, IEEE, and S. Llorente.
}

\begin{abstract}
An induction heating appliance designed to uniformly heat up metallic plates is studied in this work. It consists of one planar inductor with two concentric coils attached to a mechanism, which allows moving the inductor under the plate while heating. This system is a possible solution for the growing concept of flexible induction cooking hobs, improving their performance in flexibility and in thermal distribution in the pans. With different combinations of motion and the selective activation of the inductor coils, any pan can be uniformly heated regardless its size or position on the hob. In this paper we develop a thermal model to analyse the temperature distribution obtained in the pans for each diameter and strategy used. The model is solved using finite differences and it is validated with experimental measurements. From the calculations, the best strategy for each pan diameter is obtained.
\end{abstract}

Index Terms - Induction heating, double-coil inductor, mobile inductor, temperature distribution, thermal model.

\section{INTRODUCTION}

$\mathrm{T}$ HE induction technology has recently achieved high relevance in different heating applications due to its multiple advantages with respect to other conventional heating methods. The most extended usage takes place in the industrial thermal treatment of metallic bars or sheets, in which high power transmission with high efficiency is required. However, the induction heating is also used in domestic applications, mainly in cooking hobs, which have significantly evolved during the last years [1].

The last tendency in the current development of induction hobs is to provide to the cooktops with high flexibility regarding the position, shape and size of the pans, while obtaining a uniform heating of the pan's base. The main existing solutions (Fig. 1) contain concentric coils, enlarged coils, or multiple small coils distributed beneath the hob and supplied by different configurations of multiload power converters [2]. Once the user has placed the pan on the hob, the shape of the cooking zone is adapted to achieve a proper heating, by means of a selective activation of the inductors.

This work has been partially supported by project IPT-2011-1158-920000 from Ministerio de Economia y Competitividad, Spain, and by Departamento de Industria e Innovacion, Aragon, Spain, under Grant B132/13.

F. Sanz and C. Sagues are with the Instituto de Investigacion en Ingenieria de Aragon, Universidad de Zaragoza, 50018 Zaragoza, Spain (e-mail: fer.sanz@unizar.es; csagues@unizar.es).

S. Llorente is with the Research and Development Department, Induction Technology, Product Division Cookers, BSH Home Appliances Group, 50016 Zaragoza, Spain (e-mail: Sergio.llorente@bshg.com).
However, these solutions need a high number of inductors to ensure a good performance in flexibility, which leads to more complex inverter stages and involves higher costs. Moreover, small windings are less efficient, and the mutual magnetic coupling among inductors may cause the inverter blocks malfunctioning [3]. As well as flexibility, the users of domestic induction hobs also demand a uniform heating of the pan, especially in certain types of cooking such as griddle. When a high thermal heterogeneity appears in the bottom of the pan, the food may be overcooked in some zones, but raw in others. A similar problem also appears in certain industrial applications of induction heating, where high temperature gradients can originate cracks or other defects in the workpiece due to an irregular thermal deformation. In order to prevent this problem, some authors have proposed different solutions: with an optimal inductor design method [4], or different control methods of the power inverters in multicoil devices [5],[6],[7], the eddy current distribution induced in the workpiece is adapted to generate a homogeneous temperature distribution. Again, these proposals require using multiload inverters and a high number of coils, which affects negatively to cost and efficiency. As an alternative, the generic idea of employing mobile inductors to reduce the number of heating elements while increasing the flexibility appeared some years ago in several patents, for example in [8].

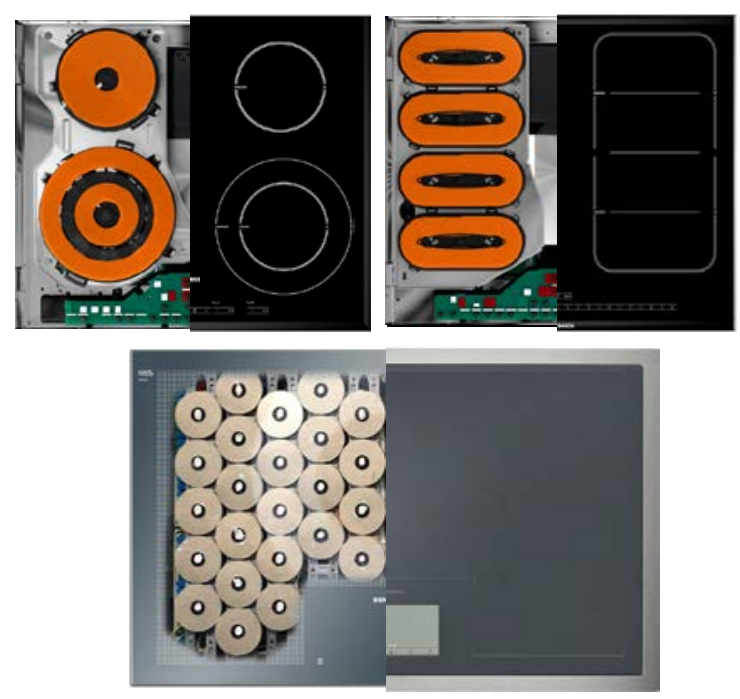

Fig. 1. Flexibility in induction hobs. Main current solutions in the cooking hobs market: concentric windings, Flex-Induction EH675MV17E (enlarged multi-inductor zone), Free-Induction CX480 (total-active surface concept). 
In this paper it is proposed an induction heating system with a double-coil inductor with concentric windings, attached to a mechanism which allows to change the position of the heating element beneath the hob surface. With the combination of different motion strategies and the selective activation of the inductor rings, the power distribution generated in the workpiece can be controlled in order to ensure a homogeneous heating. This solution with only one inductor and a simple double half-bridge resonant inverter is a cost effective alternative to the current multiwindings solutions, since less materials and components are needed for a good performance. To study this solution, a theoretical model of the heating process of metallic plates is proposed. With the model the temperature distributions obtained using different heating strategies are calculated. Thus, we determine those with which the best temperature distribution is obtained in the most common pans. The model is numerically solved using a finite differences method, which is faster than the finite element computation that other authors use [9], and gives accurate results. The calculations are validated with several experiments in a prototype. With respect to our preliminary work [10], this paper gives an extended model for the twodimensional thermal problem, and a theoretical analysis of the performance of our mobile double-inductor solution.

The paper is organized as follows: the system proposed with a double-coil, an electronics power stage and a motion system is described in Section II. A theoretical model of the induction heating process of a plate with a given power distribution is presented in Section III. The experimental validation of the thermal model is explained in Section IV. The heating performance analysis of the solution proposed, with theoretical calculations, is presented in Section V. Finally, the main conclusions are presented in Section VI.

\section{Mobile Double-Coil Induction HeAting Appliance}

The system proposed in this work consists in one circular inductor attached to a mechanism which allows to place the heating element in any position beneath the hob surface. The objective of this flexible cooking zone is to achieve a homogeneous thermal distribution when heating the most common pans (with round shape), preserving the heating efficiency as much as possible. In order to heat properly most of the pan diameters with a single mobile heating element, we propose a double-coil inductor, with two concentric windings that are fed independently. The proposed dimensions are $0.1 \mathrm{~m}$ for the internal ring and $0.15 \mathrm{~m}$ for the external ring. Combining the activation of one or both rings with the motion, it is possible to heat up any pan regardless of its size. Fig. 2 shows the proposed strategies to get a full coverage of circular pans with diameters in the range of $0.05 \mathrm{~m}$ to $0.32 \mathrm{~m}$, which are:

- Activation of the internal coil in static position centred with the pan. Adequate for the smallest pans, Fig. 2 (a).

- Activation of the internal and external coils in static position centred with the pan. Adequate for small pans, similar to the inductor diameter, Fig. 2 (b).
- Activation of the internal ring, motion with a circular trajectory. Proposed for medium-sized pans, Fig. 2 (c).

- Activation of the internal and external coils, motion with a circular trajectory. Proposed for the biggest pans, Fig. 2 (d).

Moreover, by controlling the power generated with each coil it is possible to adapt the power density distribution in the pan to improve the heating result [11]. The speed and radius of the trajectories are designed as well for each size and shape of pan.

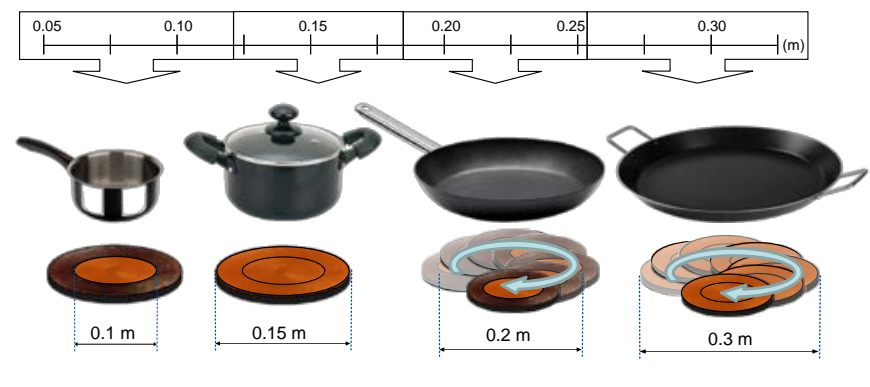

(a)

(b)

(c)

(d)

Fig. 2. Proposed heating strategies for different pan sizes. Each strategy is used in a range of pan diameters in order to heat them optimally. (a) Internal coil static. (b) Internal and external coils motionless. (c) Internal coil with circular motion. (d) Internal and external coils with circular motion.

In this work the prototype shown in Fig. 3 is used, which is based on the presented concept. The system consists of the double inductor, fed by an induction electronics stage, plus a motion system. It is entirely controlled from a PC. The motion system is a two-axes positioning mechanism controlled by an industrial PLC, which is in charge of moving the inductor beneath the hob plane. It allows setting different trajectories and adjusting the motion speed as required. The power stage contains a double half-bridge series resonant inverter with two independent outputs, one for each winding [12]. The control of the power delivered by each ring of the inductor is carried out by setting the switching frequency to a constant value and by adjusting the duty cycle of the inverter in order to reach the set-point power, [13], [14]. The selected frequencies are 60 $\mathrm{kHz}$ and $40 \mathrm{kHz}$ for the internal and external rings respectively, to avoid the audible intermodulation noise [15]. In order to ensure that the operation of the inverter is safe, the zero voltage switching (ZVS) condition is kept for high powers, and we test that the switching losses are unimportant when supplying low powers.

\section{THEORETICAL MOdEl OF TEMPERATURE Distribution}

The performance of a cooking appliance can be evaluated through the analysis of the uniformity of the temperature distribution obtained in the base of the pans during the heating process. The thermal distribution mainly depends on the power distribution generated in the pan, on the material properties, and on the geometry of the bottom of the pan. In order to study the performance of the solution proposed in this work we develop a theoretical model of the problem. With a 
two dimensional model it is possible to analyse the effects of the motion in the resultant temperature distribution in temporal and spatial dimensions. Moreover, it can be used to calculate the best heating strategy for each pan, with which a homogeneous heating is obtained, ensuring excellent cooking results.

Let be a metallic disk placed on a planar ceramic glass (Fig. 4). The disk has radius $R$ and thickness $e$. It is made of ferromagnetic steel with heat conductivity $\lambda$, specific heat capacity $c_{p p}$, and density $\rho_{p}$. A power distribution is generated with the induction heating device in the metallic disk. The glass between the inductor and the disk has thickness $e_{g}$, specific heat capacity $c_{p g}$, and density $\rho_{g}$. The disk thickness is small with respect to the other dimensions and thus it is assumed that the heat conduction in the disk occurs only radially and azimuthally. The glass temperature is equal to the disk temperature at every point due to the negligible contact resistance. The air temperature $T_{a}$ is supposed to be constant, and the heat transfer by radiation is considered linear with the temperature. Hence, a losses coefficient $h$ is defined, which includes convection and radiation heat flux in the upper side of the disk. This coefficient is estimated experimentally, and its value is assumed to be constant in the considered temperature range $\left(25-200^{\circ} \mathrm{C}\right)$. The power generated in the disk by the induction heating system is treated as a volumetric heat flux inside the disk.

The thermal model is obtained from the heat equation:

$$
P+\lambda \nabla^{2} T-\frac{h}{e}\left(T-T_{a}\right)=\rho c_{p} \frac{\partial T}{\partial t},
$$

where the first term $(P)$ is the power distribution generated by induction. The second term is the heat flux of conduction, where $\nabla^{2} T$ is the Laplacian of the disk temperature $(T)$. The third term includes the heat losses due to convection and radiation in the upper side of the disk. The last term is the variation in time of the energy stored in the system (disk and glass), thus the constant product $\rho c_{p}$ is defined as:

$$
\rho c_{p}=\frac{\rho_{p} c_{p p} e+\rho_{g} c_{p g} e_{g}}{e} .
$$

With these considerations, and using a polar coordinate system from (1), the following differential equation is obtained:

$$
\begin{gathered}
P+\lambda\left(\frac{1}{r} \frac{\partial}{\partial r}\left(r \frac{\partial T}{\partial r}\right)+\frac{1}{r^{2}} \frac{\partial^{2} T}{\partial^{2} \phi}\right)-\frac{h}{e}\left(T-T_{a}\right) \\
=\rho c_{p} \frac{\partial T}{\partial t},
\end{gathered}
$$

and two boundary conditions are defined; in $r=R$, where the radial flux is equal to the heat losses:

$$
-\left.\lambda \frac{\partial T}{\partial r}\right|_{r=R}=\left.h_{b}\left(T-T_{a}\right)\right|_{r=R},
$$

and in $r=0$, where the radial flux is null because of the geometry of the problem:

$$
-\left.\lambda \frac{\partial T}{\partial r}\right|_{r=0}=0 .
$$

The losses coefficient in the disk border $\left(h_{b}\right)$ is estimated experimentally.

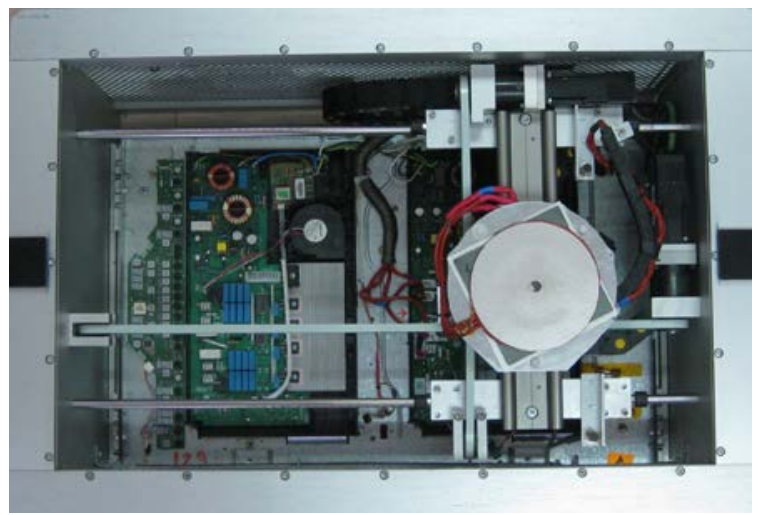

Fig. 3. Prototype of the induction heating system with a double-coil inductor attached to a two axes positioning system, and fed by an induction electronics power stage.

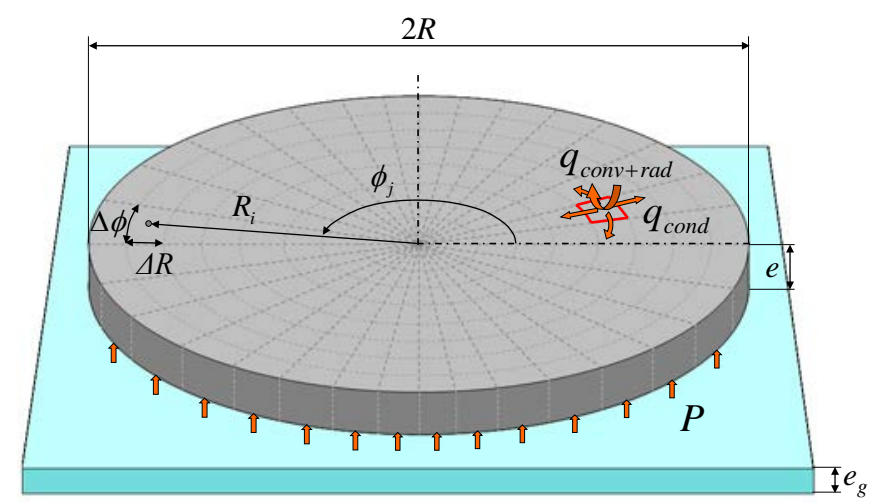

Fig. 4. Theoretical thermal model. The induction heating device transfers a magnetic power $P$ to the ferromagnetic disk. The heat generated is dissipated to the environment by convection and radiation ( $\left.\dot{q}_{c o n v+r a d}\right)$ and transmitted inside of the disk by conduction ( $\dot{q}_{\text {cond }}$ ). Each element in the grid is defined by the discrete coordinates $R_{i}, \phi_{j}$. The size of each one in radial and azimuthal directions are $\Delta R$ and $\Delta \phi$.

In order to obtain a numerical approximation to the solution of the thermal problem, the explicit finite differences method [16]-[18] with (3), (4) and (5) is used. The discrete grid shown in Fig. 4 is proposed, where the coordinates of each element $(i, j)$ are defined by $R_{i}$ and $\phi_{j}$, the element size in the radial and azimuthal directions is $\Delta R$ and $\Delta \phi$, and $\Delta t$ is the time step. The temperature of each element $T_{i, j, k}$ for every instant $k$ is the solution of the following equation system, where $n$ and $m$ are the number of elements in radial and azimuthal directions:

$(1<i<n, 1 \leq j \leq m)$

$$
\begin{gathered}
a_{i} T_{i, j, k}+b_{i}^{+} T_{i+1, j, k}+b_{i}^{-} T_{i-1, j, k}+ \\
+c_{i} T_{i, j+1, k}+c_{i} T_{i, j-1, k}=\frac{\rho c_{p}}{\Delta t} T_{i, j, k-1}+\frac{h}{e} T_{a}+P_{i, j, k},
\end{gathered}
$$




$$
\begin{aligned}
& (i=1,1 \leq j \leq m) \\
& a_{1} T_{1, j, k}+b_{1}^{+} T_{2, j, k}+c_{1} T_{1, j+1, k}+c_{1} T_{1, j-1, k}= \\
& =\frac{\rho c_{p}}{\Delta t} T_{1, j, k-1}+\frac{h}{e} T_{a}+P_{1, j, k}, \\
& (i=n, 1 \leq j \leq m) \\
& a_{n} T_{n, j, k}+b_{n}^{-} T_{n-1, j, k}+c_{n} T_{n, j+1, k}+c_{n} T_{n, j-1, k}= \\
& =\frac{\rho c_{p}}{\Delta t} T_{n, j, k-1}+\left(\frac{h}{e}+\frac{2 h_{b}}{\Delta R}+\frac{h_{b}}{R_{n}}\right) T_{a}+P_{n, j, k},
\end{aligned}
$$

where the coefficients are obtained with:

$$
\begin{gathered}
a_{i}=\frac{2 \lambda}{\Delta R^{2}}+\frac{2 \lambda}{R_{i}^{2} \Delta \phi^{2}}+\frac{\rho c_{p}}{\Delta t}+\frac{h}{e}, \\
b_{i}^{+}=-\left(\frac{\lambda}{\Delta R^{2}}+\frac{\lambda}{2 R_{i} \Delta R}\right), \\
b_{i}^{-}=-\left(\frac{\lambda}{\Delta R^{2}}-\frac{\lambda}{2 R_{i} \Delta R}\right), \\
c_{i}=-\left(\frac{\lambda}{R_{i}^{2} \Delta \phi^{2}}\right), \\
a_{1}=\frac{2 \lambda}{\Delta R^{2}}+\frac{2 \lambda}{R_{1}^{2} \Delta \phi^{2}}+\frac{\rho c_{p}}{\Delta t}+\frac{h}{e}, \\
b_{1}^{+}=-\left(\frac{2 \lambda}{\Delta R^{2}}\right), \\
c_{1}=-\left(\frac{\lambda}{R_{1}^{2} \Delta \phi^{2}}\right), \\
\frac{2 \lambda}{\Delta R^{2}}+\frac{\rho c_{p}}{R_{n}^{2} \Delta \phi^{2}}+\frac{h}{\Delta t}+\frac{2 h_{b}}{\Delta R}+\frac{h_{b}}{R_{n}}, \\
b_{n}^{-}=-\left(\frac{2 \lambda}{\Delta R^{2}}\right), \\
c_{n}=-\left(\frac{\lambda}{R_{n}^{2} \Delta \phi^{2}}\right) .
\end{gathered}
$$

The volumetric power density heating each element at the instant $k$ is $P_{i, j, k}$. It can be obtained from the power density distribution, $D p_{i, j, k}$ in $\mathrm{W} / \mathrm{m}^{2}$ as:

$$
P_{i, j, k}=\frac{D p_{i, j, k}}{e},
$$

which is directly related with the eddy current density distribution induced in the material $J_{i, j, k}$, given the electrical conductivity $\sigma$ :

$$
D p_{i, j, k}=\frac{J_{i, j, k}^{2}}{\sigma} .
$$

Other authors have proposed different methods to calculate the current and power density distributions, for example using an impedance matrix [6], [11], or with finite element computation of the electromagnetic problem [7], [9], [19]-[21]. In this work this last method is used for the calculation of the power density curve induced in a C-45 steel disk with diameter of 0.1 $\mathrm{m}$, using a circular inductor with the same size in a centred position, heating with a total power of $1 \mathrm{~W}$. In this case the power distribution is symmetrical with respect to the centre of the disk and constant in the azimuthal direction, Fig. 5 (a). To simplify the calculation of the power distribution in further situations with the mobile double-coil inductor, we consider that the shape of the distribution in the case of the single inductor can be extrapolated to the rest of cases. Thus, an example of the calculated power density distribution generated in a C-45 steel disk with diameter of $0.15 \mathrm{~m}$, using a doublecoil inductor with coils of diameters $0.1 \mathrm{~m}$ and $0.15 \mathrm{~m}$ in a centred position, with a total heating power of $1 \mathrm{~W}$ is shown in Fig. 5 (b). Moreover, in situations with the inductor placed in an off-centre position, the edge effects are omitted for simplicity.

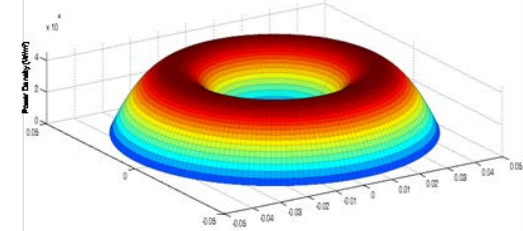

(a)

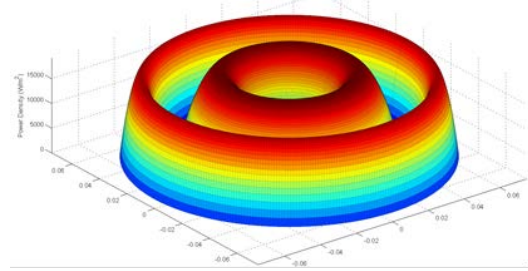

(b)
Fig. 5. Examples of the calculation of the power density distribution induced in a C-45 steel disk, based in FEM: (a) disk diameter of $0.1 \mathrm{~m}$ with an inductor of the same size, (b) disk diameter of $0.15 \mathrm{~m}$, using a double-coil inductor with diameters $0.1 \mathrm{~m}$ and $0.15 \mathrm{~m}$. The total power applied is $1 \mathrm{~W}$.

\section{MODEL VALIDATION}

The model presented in the previous section is validated by comparison of the temperature distributions obtained from the theoretical calculations and from the experimental measurement in different heating processes, using the doublecoil inductor in static position and also with motion.

The prototype presented previously (Fig. 3) is employed for heating C-45 ferromagnetic steel disks, which have a similar behaviour to the base of most circular pans. The disks have diameters between $0.07 \mathrm{~m}$ and $0.32 \mathrm{~m}$, and $0.005 \mathrm{~m}$ thickness. They are placed over a ceramic glass with thickness of 0.004 $\mathrm{m}$, specific heat capacity of $800 \mathrm{~W} \cdot \mathrm{s} \cdot \mathrm{kg}^{-1} \cdot \mathrm{K}^{-1}$ and density 2600 $\mathrm{kg} \cdot \mathrm{m}^{-3}$, which covers the inductor with a $0.001 \mathrm{~m}$ gap of air between them. An infrared camera is used to measure the temperature distribution, recording thermographic images which give the temperature values at any point of the disk. The losses coefficients $h$ and $h_{b}$ used in the calculations are estimated from the temperature measurement in different experiments. Using an optimization algorithm, the values for $h$ and $h_{b}$ which minimize the mean quadratic error between the 
calculated and measured temperatures in the considered situations are $46.5 \mathrm{~W} \cdot \mathrm{m}^{-2} \cdot \mathrm{K}^{-1}$ and $180 \mathrm{~W} \cdot \mathrm{m}^{-2} \cdot \mathrm{K}^{-1}$, respectively.

As an example of the comparison between the calculations and the measurements, the results obtained with the internal coil of the inductor in two different situations are presented. Fig. 6 shows the results of the experimental measurement and the theoretical calculation of a heating process of a C-45 steel disk of diameter $0.1 \mathrm{~m}$, placing the inductor in static position centred with the disk. The three-dimensional plots in the left side are, from bottom to top, the calculated power density distribution generated in the disk, the calculated temperatures, and the temperature distribution measured with the infrared camera, after $57 \mathrm{~s}$ applying $580 \mathrm{~W}$. The plot in the top-right corner shows the measured and calculated temperature profiles along a radius of the disk after $57 \mathrm{~s}$. The plot in the bottomright corner shows the temperature evolution in the centre of the disk and in a point at $0.025 \mathrm{~m}$ from the centre. The results show a good agreement between the experimental and theoretical data in the spatial distribution as well as in the temporal evolution of the temperature. On the other hand, Fig. 7 shows the results of heating a disk of $0.2 \mathrm{~m}$ of diameter with the inductor following a circular trajectory of $0.06 \mathrm{~m}$ of radius, with speed of $1.11 \mathrm{rad} / \mathrm{s}$, applying $1200 \mathrm{~W}$ during $108 \mathrm{~s}$. Again, the model shows a good approximation of the spatial distribution calculation as well as of the temporal evolution. Thus, the model correctness can be confirmed. The small deviation between the theoretical calculation and the experimental measurement may be a consequence of the errors in the estimation of the power density distribution and also of the simplifications considered in the modelling.

\section{Theoretical Study of the Heating Performance}

The worst operation case in an induction cooktop is the preheating process applying a high power level, which is commonly employed by users to obtain a fast heating of the pan before adding the food. At the end of this process, when a maximum temperature of around $180^{\circ} \mathrm{C}$ has been reached, the temperature distribution is more heterogeneous, due to the negligible effect of the heat diffusion through the base of the pan with respect to the heat generation.

As aforementioned, the performance in thermal distribution of the mobile double-coil heating appliance is analysed using the theoretical model proposed. For this purpose we simulate the pre-heating process of circular pans with diameters in the range of $0.05 \mathrm{~m}$ to $0.32 \mathrm{~m}$, using the heating strategies with the mobile inductor that were proposed previously (Fig. 2). Then the maximum temperature differences for each disk size and strategy are calculated. Thus, the best solution for each range of pan diameters is obtained, with which the temperature differences are lesser and a more uniform heating is achieved. The average power density used in the pre-heating process in induction cooking hobs is generally lower for big pans than for the small pans. Hence, we use three different average power densities for three ranges of disk diameters, shown in Table I.
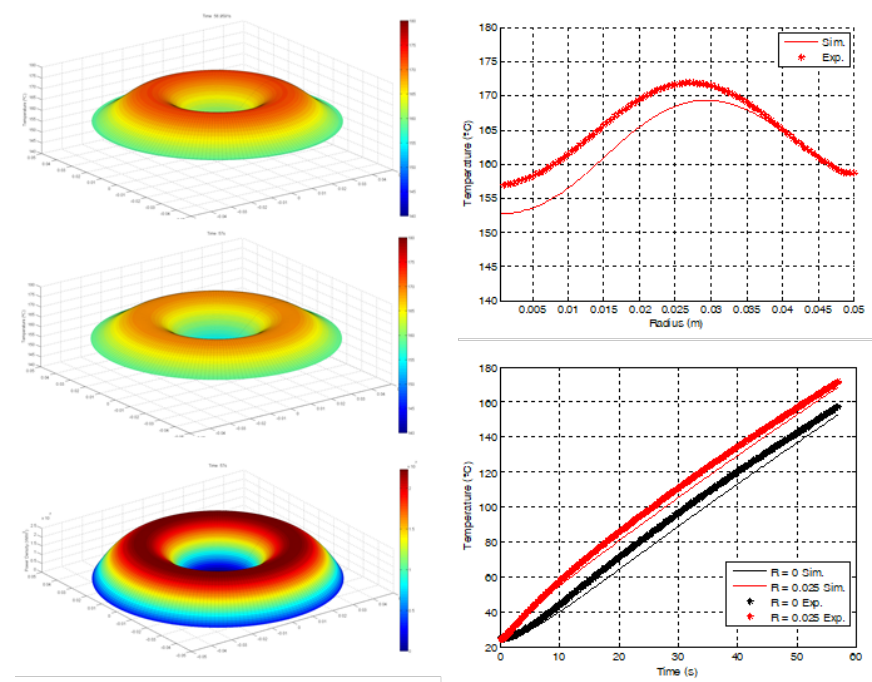

Fig. 6. Comparison of calculated and measured temperatures after applying $580 \mathrm{~W}$ during $57 \mathrm{~s}$ in a disk of diameter $0.10 \mathrm{~m}$ of C-45 steel, using a circular inductor of the same diameter, centred with the disk. In the left side, from bottom to top are shown the calculated power density distribution generated in the disk, the calculated temperatures and the measured temperatures after $57 \mathrm{~s}$. In the right side, the top plot shows the temperature profiles along a radius and the bottom plot shows the temperature evolution on two points at different distances to the centre.
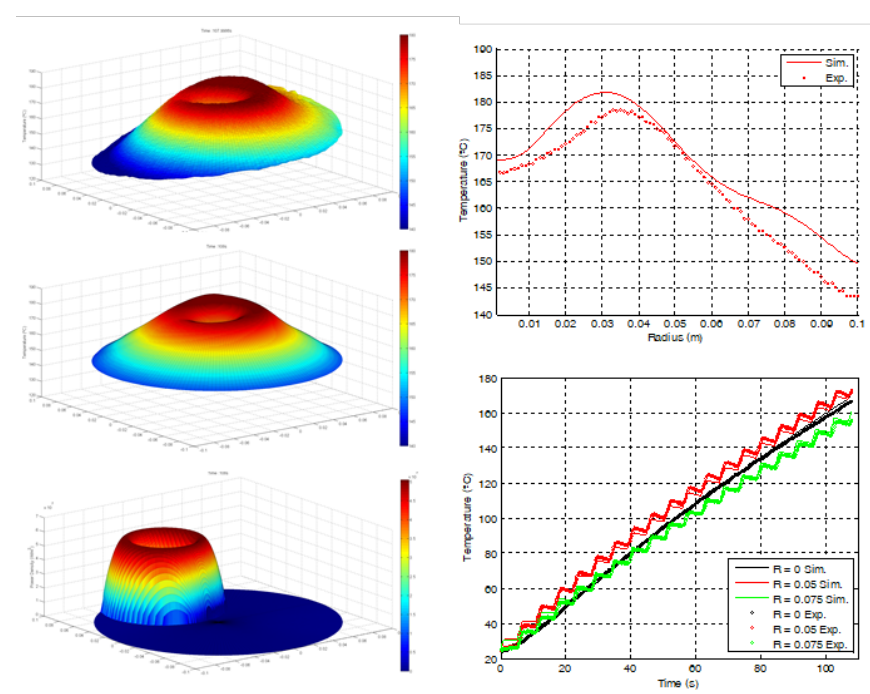

Fig. 7. Comparison of calculated and measured temperatures after applying $1200 \mathrm{~W}$ during $108 \mathrm{~s}$ in a disk of diameter $0.2 \mathrm{~m}$ of C-45 steel, using a circular inductor of diameter $0.1 \mathrm{~m}$, following a circular trajectory of radius $0.06 \mathrm{~m}$ and speed $1.11 \mathrm{rad} / \mathrm{s}$. In the left side, from bottom to top are shown the calculated power density distribution generated in the disk, the calculated temperatures and the measured temperatures after $108 \mathrm{~s}$. In the right side, the top plot shows the temperature profiles along a radius and the bottom plot shows the temperature evolution on three points at different distances to the centre.

The results obtained from the simulation of the strategies with the inductor placed in a static position, centred with the pan with one or both coils of the inductor activated, are shown in Fig. 8. These strategies are proposed for the smallest pans, with diameters from $0.05 \mathrm{~m}$ to $0.18 \mathrm{~m}$. The plot shows the maximum temperature differences, calculated and measured, when $180^{\circ} \mathrm{C}$ are reached during the pre-heating process for different disk sizes. From these results the best activation 
strategy of the coils for each pan size can be derived, corresponding to that which produces the lowest temperature difference. Hence, for pan diameters between $0.05 \mathrm{~m}$ and 0.12 $\mathrm{m}$ the best strategy is the activation of the internal coil, and from $0.12 \mathrm{~m}$ to $0.17 \mathrm{~m}$ it is more suitable to activate both coils, generating the same amount of power with each one (power division between coils 50\% / 50\%). With pans over $0.17 \mathrm{~m}$, the heating of the central area of the base is more significant than in the peripheral area, obtaining higher temperature differences and therefore an unsuitable heating result.

TABLE I

POWER DENSITY LEVELS

\begin{tabular}{cc}
\hline \multicolumn{2}{c}{ POWER DENSITY LEVELS } \\
\hline Power Density $\left(\mathrm{W} / \mathrm{m}^{2}\right)$ & Disk Diameter Range $(\mathrm{m})$ \\
\hline $8 \mathrm{e} 4$ & 0.05 to 0.11 \\
$4 \mathrm{e} 4$ & 0.11 to 0.21 \\
$2.6 \mathrm{e} 4$ & 0.21 to 0.32 \\
\hline \hline
\end{tabular}

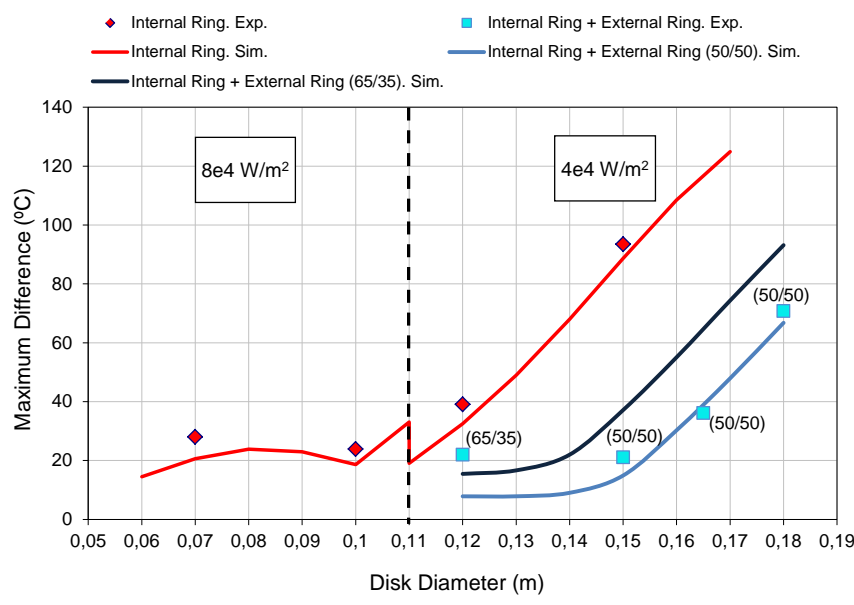

Fig. 8. Maximum temperature differences calculated and measured in a preheating process of C-45 steel disks, using strategies with the double inductor in a static position centred with the disk, with one or both coils of the inductor activated. In the case of the activation of both coils, two different power divisions between coils have been considered, in simulation and in the experiments.

The simulation results in the case of heating strategies with the inductor describing circular trajectories beneath the pan, with one coil and with both coils activated, are presented in Fig. 9. These strategies are proposed for medium sized pans and the largest pans, with diameters from $0.16 \mathrm{~m}$ to $0.32 \mathrm{~m}$. The best heating strategy for each pan diameter can also be established from the calculation results. With a total coverage of the inductor (dashed line), the heating efficiency is maximum, however with the smallest pan diameters an overlap of the magnetic field occurs in the centre of the base, which generates a hot spot and hence an uneven temperature distribution (Fig. 10 (a)). In order to obtain a more uniform thermal distribution, the trajectory radius can be selected for each pan, even if the inductor is partially uncovered during the heating process (Fig. 10 (b)). Therefore, pans with diameters between $0.16 \mathrm{~m}$ and $0.24 \mathrm{~m}$ are heated with a more uniform thermal distribution with the internal coil and a circular motion, selecting an appropriate trajectory radius. The biggest pans, with diameters between $0.25 \mathrm{~m}$ and $0.32 \mathrm{~m}$, are heated more uniformly with both coils activated plus the motion. In this case, for diameters between $0.25 \mathrm{~m}$ and $0.27 \mathrm{~m}$ the results are improved if the internal ring generates a higher power than the external ring, for example with a power division between coils $65 \%$ / 35\%.

- Internal Ring + Motion. Exp.

- Internal Ring + External Ring + Motion. Exp.

- - - Internal Ring + Motion: Total coverage. Sim.

_ Internal Ring + Motion: Improved distribution. Sim.

_Internal Ring + External Ring (50/50)+ Motion: Improved distribution. Sim.

__ Internal Ring + External Ring (65/35)+ Motion: Improved distribution. Sim.

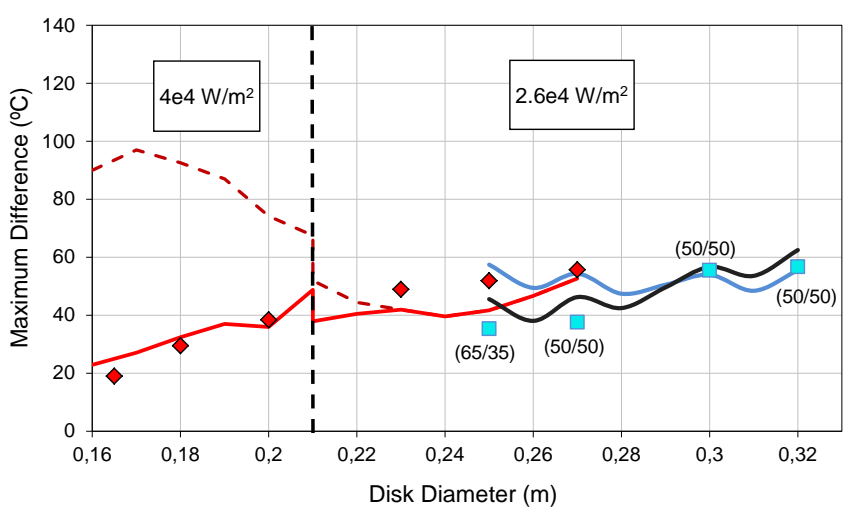

Fig. 9. Maximum temperature differences calculated and measured in a preheating process of $\mathrm{C}-45$ steel disks, using strategies with the double inductor following circular trajectories beneath the disk, with one or both coils of the inductor activated. In the case of the activation of both coils, two different power divisions between coils have been considered.

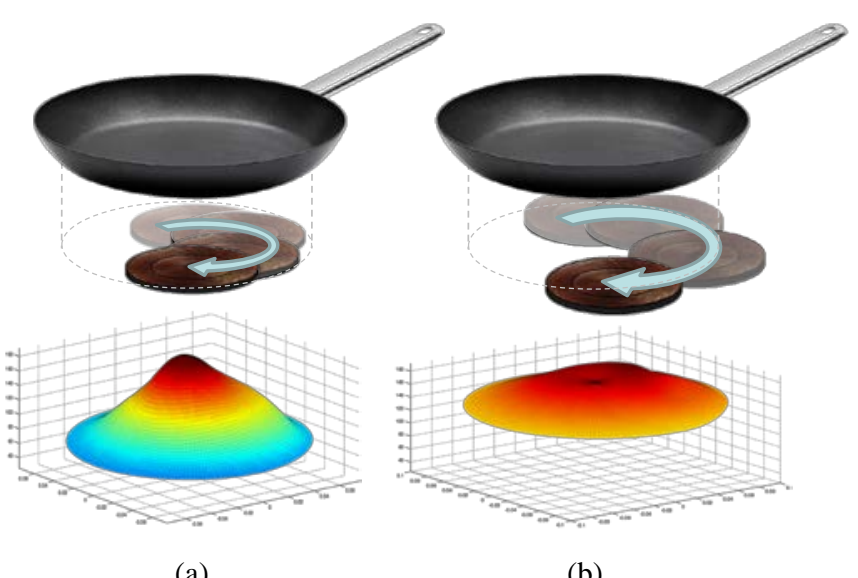

(a)

(b)

Fig. 10. Effect of the magnetic field overlap in the centre of the pan over the temperature distribution, depending on the circular trajectory radius: (a) radius selection to guarantee a total coverage of the inductor, maximum efficiency, (b) radius selection to partially uncover the inductor, improved thermal distribution.

\section{CONCLUSION}

In this paper, an induction heating system with mobile inductors for domestic induction hobs has been presented. The evaluation of the flexible induction heating appliance with a mobile double-coil inductor has been carried out with a theoretical model of the heating process, as well as the study 
of optimal strategies combining inductors with different diameters and motion. Attending to the performance when heating up different pans of any size, we have proved that excellent results are obtained with the proposed solution. The temperature differences obtained from experimental measurements and theoretical calculations in several preheating processes of steel disks with different sizes are very low. Hence, in normal cooking conditions, the temperature homogeneity is ensured, obtaining a uniform cooking of the food.

\section{REFERENCES}

[1] J. Acero, et al., "Domestic Induction Appliances: An overview of recent research,” IEEE Industry Applications Magazine, Vol. 16, No. 2, pp. 3947, March/April 2010.

[2] O. Lucía, C. Carretero, J. M. Burdío, J. Acero, and F. Almazán, "Multiple output resonant matrix converter for multiple induction heaters," IEEE Transactions on Industry Applications, Vol. 48, No. 4, pp. 1387-1396, July/August 2012.

[3] A. M. Green, "Operation of inverters supplying mutually coupled induction heating loads," IEEE Colloquium on Electromagnetics and Induction Heating, no. 264, Vol. 4, pp. 1-3, 1996.

[4] Jin-kyu Byun, Kyung Choi, Hee-Succ Roh, and Song-Yop Hahn, "Optimal design procedure for a practical induction heating cooker," IEEE Transactions on Magnetics, Vol. 36, No. 4, pp. 1390-1393, July 2000.

[5] H. N. Pham, H. Fujita, K. Ozaki, and N. Uchida, "Dynamic analysis and control for resonant currents in a zone-control induction heating system,” IEEE Transactions on Power Electronics, Vol. 28, No. 3, pp. 1297-1307, March 2013.

[6] H. N. Pham, H. Fujita, K. Ozaki, and N. Uchida, "Estimating method of heat distribution using 3-D resistance matrix for zone-control induction heating systems," IEEE Transactions on Power Electronics, Vol. 27, No. 7, pp. 3374-3382, July 2012.

[7] J. Egalon, S. Caux, P. Maussion, M. Souley, O. Pateau, "Multiphase system for metal disc induction heating: modeling and RMS current control," IEEE Transactions on Industry Applications, Vol. 48, No. 5, pp. 1692-1699, September / October 2012.

[8] J. Acero, R. Alonso, M. A. Buñuel, D. Casanova, J. R. García, I. Garde, A. Mediano, F. Monterde, and C. Pina. "Hob with a movable heating element.” World Patent WO2008EP51013, August 28, 2008.

[9] M. Souley, P. Maussion, P. Ladoux, O. Pateau, "Simplified model of a metal disc induction heating system," International Symposium on Heating by Electromagnetic Sources, pp. 137-144, May 2010.

[10] F. Sanz, C. Franco, C. Sagües, D. Paesa, and S. Llorente, "Flexible cooking zone with 2D mobile inductors for cooking hobs”, 38th Annual Conference on IEEE Industrial Electronics Society, pp. 3262-3267, October 2012.

[11] J. Acero, C. Carretero, I. Millán, O. Lucía, R. Alonso, and J. M. Burdío, "Analysis and modelling of planar concentric windings forming adaptable-diameter burners for induction heating appliances,” IEEE Transactions on Power Electronics, vol. 26, no. 5, pp. 1546-1558, May 2011.

[12] C. Carretero, O. Lucia, J. Acero and J. M. Burdio, "Computational modeling of two partly coupled coils supplied by a double half-bridge resonant inverter for induction heating appliances," IEEE Transactions on Industrial Electronics, Vol 60, No. 8, pp. 3092-3105, August 2013.

[13] O. Lucía, J. M. Burdio, I. Millan, J. Acero, and L. A. Barragan, "Efficiency-oriented design of ZVS half-bridge series resonant inverter with variable frequency duty cycle control," IEEE Transactions on Power Electronics, vol. 25, no. 7, pp. 1671-1674, July 2010.

[14] A. Cataliotti, F. Genduso, A. Raciti, and G. R. Galluzzo, "Generalized PWM-VSI control algorithm based on a universal duty-cycle expression: theoretical analysis, simulation results, and experimental validations," IEEE Transactions on Industrial Electronics, vol. 54, no. 3, pp. 1569-1580, June 2007.

[15] J. M. Burdío, L. A. Barragán, P. Hernandez, A. Lorente, F. Monterde, and S. Llorente. "Method for avoiding or reducing noise interference in a converter circuit with multiple simultaneously operated outputs.” World Patent WO2005043737, May 12, 2005.
[16] W. Mai and G. Henneberger, "Calculation of the transient temperature distribution in a TFIH device using the impedance boundary condition," IEEE Transactions on Magnetics, Vol. 34, No. 5, pp. 3094-3097, 1998.

[17] M. Kaminski, "The use of the finite differences method to solve nonlinear equation of thermal conduction," Proceedings of the International Conference Modern Problems of Radio Engineering, Telecommunications and Computer Science, pp. 52-55, Feb. 2004.

[18] M. Clemens, E. Gjonaj, P. Pinder, and T. Weiland, "Numerical simulation of coupled transient thermal and electromagnetic fields with the finite integration method," IEEE Transactions on Magnetics, Vol. 36, No. 4, pp. 1448-1452, July 2000.

[19] C. Chaboudez, S. Clain, R. Glardon, D. Mari, J. Rappaz, and M. Swierkosz, "Numerical modeling in induction heating for axisymmetric geometries," IEEE Transactions on Magnetics, Vol. 33, No. 1, pp. 739745, 1997.

[20] E. J. W. ter Maten, J. B. M. Melissen, "Simulation of inductive heating," IEEE Transactions on Magnetics, Vol. 28, No. 2, pp. 1287-1290, 1992.

[21] Koichi Ishida, Toshiya Itaya, Akio Tanaka, Nobuo Takehira, "Magnetic field analysis of an arbitrary shaped coil using shape functions," IEEE Transactions on Magnetics, Vol. 45, No. 1, pp. 104-112, January 2009.

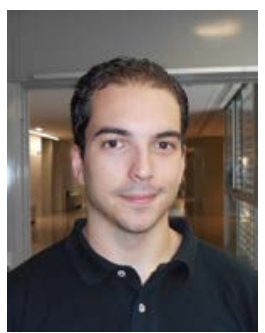

Fernando Sanz (S'12) received the M.Sc. degree in Informatics and Systems Engineering from the University of Zaragoza, Zaragoza, Spain.

Since 2012, he has been with the Aragon Institute for Engineering Research (I3A) and with the Departamento de Informatica e Ingenieria de Sistemas of the University of Zaragoza, where he is currently working on his $\mathrm{PhD}$. He has been working on induction heating modelling and on energy-saving domestic appliances. His research interests include the modelling and control of heat transfer processes and induction heating systems.

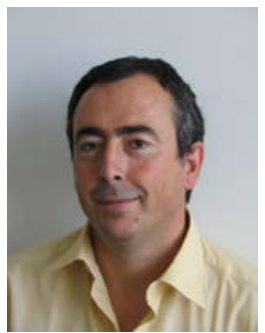

Carlos Sagues (M'00-SM'11) received the M.Sc. and Ph.D. degrees from the University of Zaragoza, Zaragoza, Spain.

He was engaged in research on force and infrared sensors for robots during the Ph.D. degree. Since 1994, he has been an Associate Professor and, since 2009, a Full Professor with the Departamento de Informatica e Ingenieria de Sistemas, University of Zaragoza, where he has also been the Head Teacher. His current research interests include control systems and industry applications, computer vision, visual control, and multivehicle cooperative control.

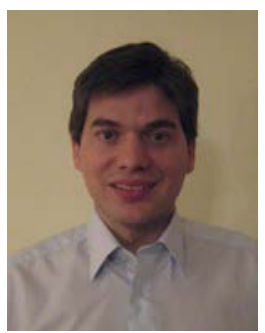

Sergio Llorente received the M.Sc. from the University of Zaragoza, Zaragoza, Spain.

In 2001 he joined BSH Bosch Siemens Home Appliances. He has held different positions in the Research and Development Department of Induction Cooktops, Zaragoza, Spain. He is currently in charge of several research lines and pre-projects, and he is inventor in more than 150 patents. He also has been an Assistant Professor at the University of Zaragoza since 2004. His interests include power electronics, simulation and control algorithms for power electronics and temperature control. 